\title{
LA PESTE PORCINE AFRICAINE (PPA) EN CHINE, EFFETS SUR LA PRODUCTION PORCINE ET LES FLUX D'IMPORTATIONS, DANS LE CONTEXTE INSTITUTIONNEL ET ÉCONOMIQUE
}

\author{
AFRICAN SWINE FEVER (ASF) IN CHINA: CONSEQUENCES ON PIG \\ PRODUCTION AND IMPORT FLOWS, IN THE INSTITUTIONAL AND \\ ECONOMIC CONTEXT
}

Par Jean-Marc CHAUMET ${ }^{l}$

(Communication présentée le 3 février 2021, manuscrit accepté le 20 septembre 2021)

Mots-clés : Chine, viande porcine, commerce, politique agricole, politique environnementale, PPA

Keywords: China, pig meat, trade, agricultural policy, environmental policy, ASF

\section{INTRODUCTION}

Apparue il y a deux ans et demi dans le pays, la PPA a montré aux décideurs chinois l'impossibilité, en temps de crise, de poursuivre plusieurs objectifs politiques à la fois et donc mis en évidence la fragilité du système alimentaire chinois. Elle a en effet placé les autorités face à au dilemme de choisir entre limiter l'inflation, conserver un niveau élevé d'autosuffisance en viande porcine et appliquer une nouvelle politique environnementale.

\section{UNE HAUSSE DES COURS DU PORC QUI PEUT FRAGILISER LA STABILITÉ SOCIALE}

La Peste Porcine Africaine (PPA) est apparue dans le nord-est de la Chine au $2^{\text {ème }}$ semestre 2018, avant de se répandre dans tout le pays. Les conséquences sur le secteur porcin chinois ont été dramatiques avec une chute de près de $40 \%$ du cheptel en un peu plus d'un an et de plus de $21 \%$ de la production nationale, d'après les données officielles (China Statistical Yearbook, 2020). Cette réduction drastique de l'offre domestique a entraîné une multiplication par plus de trois des prix de la viande sur les marchés à travers le pays entre février 2019 et février 2020. Le record a été établi à $59 \mathrm{RMB} / \mathrm{kg}$ (soit environ 9 $€ / \mathrm{kg}$ de viande). Or la viande porcine est emblématique de la cuisine chinoise et représentait près des deux tiers de la consom- mation de viande en Chine en 2018, soit près de $40 \mathrm{~kg}$ équivalent carcasse annuels (Abcis, 2019). Elle occupe donc une place importante dans la vie des Chinois, dans le panier de la ménagère et conséquemment dans le calcul de l'indice des prix à la consommation. Elle pèserait 13\% dans l'indice des prix à la consommation alimentaire et 3\% dans l'indice total des prix à la consommation. Les prix du porc ont tiré l'indice des prix alimentaires à des niveaux proches de ceux de 2008. Si la Chine ne craint plus l'apparition de famines sur son sol, les efforts dans le cadre de sa politique de sécurité alimentaire se sont déplacés vers la lutte contre l'inflation, dans un objectif de stabilité sociale dans un pays où les contestations sont rarement autorisées.

\section{DES IMPORTATIONS QUI ACCENTUENT LA DÉPENDANCE ALIMENTAIRE CHINOISE}

Pour tenter de juguler l'inflation et de satisfaire la demande des consommateurs, la Chine a procédé à des importations massives de viandes et de produits porcins, en atteignant des records historiques. En 2020, les volumes importés ont atteint 5,7 millions de tonnes, une multiplication par 2,7 entre 2018 et 2020. La PPA a également été indirectement à l'origine d'une hausse de la consommation et d'importations d'autres viandes (volaille, bœuf), creusant encore plus le déficit commercial agro-alimentaire chinois. Les importations massives de viande porcine ont donc mis à mal la relative autosuffisance en viande porcine. Si la sécurité alimentaire se traduit souvent en chinois

(1) Jean-Marc Chaumet, Agroéconomiste à IDELE et ABCIS, 149 Rue de Bercy, 75595, Paris Cedex 12.

Courriel : jean-marc.chaumet@idele.fr 
par « sécurité des grains " (Chaumet, 2020), la viande porcine fait partie des produits alimentaires au centre de la politique agricole du pays. Alors que le virus circule encore dans les exploitations chinoises, dépendre de l'étranger pour l'approvisionnement de la première viande consommée dans le pays est ainsi considéré comme une faiblesse, d'autant plus que cette situation peut s'avérer dangereuse sur un plan géoéconomique. Or la dépendance alimentaire est considérée comme un talon d'Achille pour un pays qui veut devenir la lère puissance mondiale en 2049 (Chaumet \& Pouch, 2017). En outre, la dépendance vis-à-vis de son grand adversaire, les États-Unis, constitue un risque géopolitique accru notamment avec l'accord commercial signé en janvier 2020. Les États-Unis sont devenus le deuxième fournisseur de viande porcine de la Chine fin 2020. Pour tenter de limiter la dépendance envers quelques pays mais également envers des entreprises étrangères, le nouveau plan pour la filière porcine encourage les grandes entreprises de production chinoises à créer des élevages de porcs à l'étranger, afin de combler le manque de porcs sur le marché intérieur. La construction des élevages de porcs à l'étranger ne serait possible que dans les pays ayant des relations commerciales bilatérales stables avec la Chine, et indemnes de la peste porcine africaine (Husson, 2020). Mais les décideurs chinois sont bien conscients que le marché international est insuffisant pour nourrir la Chine, en céréales tout comme en porc. La seule solution à moyen terme est donc de redevenir autosuffisant en porc (Bloomberg News, 2020). Pour atteindre cet objectif, le gouvernement central chinois accentue la pression sur les autorités locales afin que ces dernières mettent en ouvre des plans de reconstitution du cheptel porcin. Ces actions se mettent en place au niveau local à travers des programmes de subventions et la facilitation de construction de nouvelles exploitations, notamment sur des terres normalement dévolues à des cultures de céréales (Yang, 2019).

\section{UNE POLITIQUE ENVIRONNEMENTALE REVUE}

Mais afin de recouvrer au plus vite l'autosuffisance nationale, les autorités chinoises ont été contraintes de revenir, partiellement, sur leurs objectifs environnementaux décidés quelques années auparavant. En 2013, un rapport officiel pointe une nouvelle source de pollution devenue prépondérante : les déjections animales (Chaumet, 2020). Elles seraient responsables de près de $60 \%$ des rejets agricoles de phosphore et de près de $40 \%$ de ceux d'azote. Les exploitations de grande taille sont montrées du doigt pour leur forte contribution à ce phénomène. Une des mesures phares prise alors dans la lutte contre les pollutions d'origine animales a consisté à délimiter des zones sans élevage qui englobent des périmètres habités ou comprenant des sources d'eau potable ou des zones environnementalement fragiles ou des zones touristiques. En décembre 2017, les zones interdites totalisaient au niveau national $630000 \mathrm{~km}^{2}$, soit une superficie supérieure à celle de la France continentale, et plus de 200000 élevages avaient été fermés ou déplacés. Cette mesure a été complétée par une taxe sur les pollutions environnementales qui vise les pollutions de l'air, de l'eau et du sol. Ainsi, après avoir limité l'élevage dans de nombreuses zones considérées comme vulnérables et déplacé une partie de la production porcine, les consignes de Pékin ont été de faciliter la création d'exploitations partout où cela était nécessaire, quitte à oublier ces nouvelles règles environnementales. En mars 2020, le ministère chinois de l'Environnement a ainsi simplifié les exigences d'enregistrement environnemental pour l'industrie de l'élevage porcin et assoupli l'interdiction d'élevage porcin dans 14000 zones auparavant interdites d'élevage (Xinhua News Agency, 2020). Le Ministère de l'Environnement a notamment mentionné que "La protection de l'environnement ne devrait pas être utilisée comme excuse pour freiner l'élevage porcin ».

\section{CONCLUSION}

Les récents évènements ont donc forcé les dirigeants chinois à faire des choix, ne pouvant mener de fronts leurs différentes politiques visant à atteindre une autosuffisance en porcs, un prix du porc modéré et une large protection géographique contre les pollutions animales. Les choix d'une inflation limitée à court terme et d'une autosuffisance retrouvée à moyen terme ont ainsi été érigés en priorité.

\section{BIBLIOGRAPHIE}

- ABCIS. La crise sanitaire en Chine bouleverse la filière porcine chinoise et l'équilibre des marchés mondiaux du porc, 2019. $90 \mathrm{pp}$.

- Bloomberg New: China wants to rely almost entirely on pork produced at home, 2020. Disponible à : https://www.bnnbloomberg.ca/chin a-wants-to-rely-almost-entirelyon-pork-produced-at-home-1.1500312 (consulté le 03.05.2021)

- Chaumet J-M, Pouch T. La Chine au risque de la dépendance alimentaire. Presses Universitaires de Rennes ; 2017.

- Chaumet J-M. La politique agricole chinoise : changement de cap pour un même objectif. Économie rurale. 2020 ; $372: 153-170$

- China Statistical Yearbook, différentes années. Disponible à : http://www.sta ts.gov.cn/english/Statisticaldata/AnnualData/ (consulté le 03.05.2021)

- Husson E. Le Covid impacte le marché chinois du porc. La Lettre Chine_ABCIS. Mai 2020. Disponible à : https://abcis.com/la-lettre-chine-abcis/ (consulté le 03.05.2021)

- Husson E, Chaumet JM. Importations chinoises record de produits porcins au printemps 2020. La Lettre Chine_AB-
CIS. Juillet 2020. Disponible à https://abcis.com/la-lettre-chine-abcis/ (consulté le 03.05.2021)

- Xinhua News Agency. China eases unlawful restrictions on hog production. 2020. Disponible à : http://www.xinhuanet.com/english 2020-03/10/c 138863746.htm (consulté le 03.05.2021)

- Yang W. Land use changes to lift hog production. 2019. Disponible à : http://english.www.gov.cn/statecou ncil/ministries/201912/20/content WS5dfc0620c6d0bcf8c4c19271.html (consulté le 03.05.2021) 